\title{
Research on Shape Remain of Near-Earth Radial Collinear 4-Craft Coulomb Formation
}

\author{
SUN Yunlong , YUAN Changqing and LI Zhengguang \\ 2222 Nanhu Road, Changchun City, Jilin Province, China
}

\begin{abstract}
This paper investigates the non-linear relative kinetic model of the collinear 4-craft Coulomb formation in geostationary orbit. Considering the fact that the formation remains statically fixed in the radial direction, using the equilibrium conditions to linearize the dynamic model. To keep the radial static stability of the collinear 4-craft Coulomb formation, the LQR controller is designed based on the linear dynamic model, taking the Coulomb force as the only control force. Considering the unmodeled disturbance force and the model error, the improved LQR control law is designed to improve the robustness of the traditional LQR controller. Simulation is executed by Matlab/Simulink, and numerical simulation results demonstrate the effectiveness of the dynamic model and the proposed control strategy.
\end{abstract}

\section{Introduction}

Satellites formation flying has obvious advantages in synthetic aperture radar and Spatial Interferometer. For recent years, utilizing Coulomb force to control satellite formation is a hot topic. The basic idea of Coulomb propulsion of free-flying vehicles is to control the spacecraft formation shape and size using the inter-spacecraft forces. This propellant-less thrusting is an attractive solution over conventional electric propulsion or chemical thrusting. It has very little electrical power requirements (one Watt or less) with a renewable energy source. And it causes no thruster plume contamination of the neighboring spacecraft, which increases the lifetime of the equipment and the probability of mission success.

Parker and King introduced the Coulomb propulsion concept in References 1 and 2 in 2002. It was proposed to control a cluster of free-flying spacecraft. Natarajan ${ }^{[3-5]}$ designed feedback controller for 2-craft Coulomb formation at GEO based on relative distance and attitude dynamics equations. Inampudi and Schaub ${ }^{[6-7]}$ designed charge feedback controller for 2-craft Coulomb formation at libration point based on dynamics equations.

\section{Dynamics model}

A 4-craft Coulomb formation is considered as shown in the Figure 1. It is assumed that the center of mass spin around the earth in GEO and the four charged satellites are nominally aligned along the radial direction with initial angle and separate distance error. In order to describe the relative motion of the satellite with respect to the formation center of mass, a rotating Hill orbit frame
$\boldsymbol{H}:\left\{\boldsymbol{O}_{\boldsymbol{r}}, \boldsymbol{O}_{\boldsymbol{\theta}}, \boldsymbol{O}_{\boldsymbol{h}}\right\}$ is chosen as shown in Figure 1. The relative position vector of the satellite is defined as $\boldsymbol{\rho}_{i}=\left[\begin{array}{lll}x_{i} & y_{i} & z_{i}\end{array}\right]^{T}$.

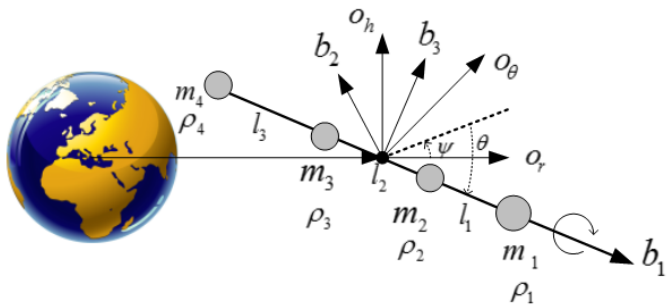

Figure 1. 4-craft Coulomb formation

The center of mass condition is defined as:

$$
m_{1} \boldsymbol{\rho}_{1}+m_{2} \boldsymbol{\rho}_{2}+m_{3} \boldsymbol{\rho}_{3}+m_{4} \boldsymbol{\rho}_{4}=0
$$

Where $m_{i}$ is the $i^{\text {th }}$ satellite mass.

Consider a coordinate frame $\boldsymbol{B}:\left\{\boldsymbol{b}_{1}, \boldsymbol{b}_{2}, \boldsymbol{b}_{3}\right\}$ as shown in Figure 1.l. is the relative distance between satellites. The relationship between $\rho_{i}$ and $l_{i}$ can be expressed by:

$$
\left\{\begin{array}{l}
\rho_{1}-\rho_{2}=l_{1} \\
\rho_{2}-\rho_{3}=l_{2} \\
\rho_{3}-\rho_{4}=l_{3}
\end{array}\right.
$$

National Natural Science Foundation of China (11372353) 
The direction cosine matrix that relates the $\boldsymbol{B}$ frame to $\boldsymbol{H}$ frame is given as $[\boldsymbol{B} \boldsymbol{H}(\theta, \psi)]$.Using Eq. (1) (2) and $[\boldsymbol{B H}(\theta, \psi)]$, the position vector ${ }^{H} \boldsymbol{\rho}_{i}$ can be solved. Take a derivative with respect to ${ }^{H} \boldsymbol{\rho}_{i}$, we can get speed vector ${ }^{H} \dot{\boldsymbol{\rho}}_{i}=\left[\begin{array}{ccc}\dot{x}_{i} & \dot{y}_{i} & \dot{z}_{i}\end{array}\right]^{T}$. The inertial velocity expressed in the $\boldsymbol{H}$ frame becomes

$$
\boldsymbol{v}_{i}=\left(\begin{array}{c}
\dot{x}_{i}-\Omega y_{i} \\
\dot{y}_{i}+\Omega\left(x_{i}+r_{c}\right) \\
\dot{z}_{i}
\end{array}\right)
$$

The kinetic energy of the formation is: The kinetic energy of the formation is: $\boldsymbol{T}=\frac{1}{2} \sum_{i=1}^{4} m_{i}\left\|\boldsymbol{v}_{i}\right\|^{2}$.Using ${ }^{H} \boldsymbol{\rho}_{i}$ 、 ${ }^{H} \dot{\boldsymbol{\rho}}_{i}$ and Eq. (3), the $\boldsymbol{T}$ is rewritten as

$$
T=\frac{1}{2 \bar{m}}\left[\dot{\mathrm{X}}+\mathrm{X}\left(\dot{\theta}^{2}+(\dot{\psi}+\Omega)^{2} \cos ^{2} \theta\right)\right]+\frac{\bar{m}}{2} \Omega^{2} \boldsymbol{r}_{c}{ }^{2}
$$

Where $\mathrm{X}=a_{1} l_{1}^{2}+a_{2} l_{2}^{2}+a_{3} l_{3}^{2}+2 a_{4} l_{1} l_{2}+2 a_{5} l_{1} l_{3}+2 a_{6} l_{2} l_{3}$, $a_{1}=m_{1}\left(m_{2}+m_{3}+m_{4}\right), a_{2}=\left(m_{1}+m_{2}\right)\left(m_{3}+m_{4}\right)$,

$a_{3}=\left(m_{1}+m_{2}+m_{3}\right) m_{4}, a_{4}=m_{1}\left(m_{3}+m_{4}\right)$,

$a_{5}=m_{1} m_{4}, a_{6}=\left(m_{1}+m_{2}\right) m_{4}, \bar{m}=\sum_{i=1}^{4} m_{i}$.

The gravitational potential energy of the formation is:

$$
V_{g}=-\frac{\mu \bar{m}}{r_{c}}-\frac{\mu \mathrm{X}}{2 \bar{m} r_{c}^{3}}\left(3(\cos \theta \cos \psi)^{2}-1\right)
$$

The Coulomb potential for the two-craft formation is:

$$
V_{e}=k_{c} \sum_{i=1}^{3} q_{i} \sum_{j=i+1}^{4} \frac{q_{j}}{L} e^{-L / \lambda_{d}}
$$

Where $L$ is the distance between the two satellites. $\theta$ and $\psi$ are the roll angle out of the orbital plane and the pitch angle in the orbital plane. $q_{i}$ is the $i^{\text {th }}$ satellite charge.

The equations of motion are deduced from the Lagrangian function $\boldsymbol{L}=T-\left(V_{g}+V_{e}\right)$ in the following form:

$$
\frac{d}{d t} \frac{\partial \boldsymbol{L}}{\partial \dot{q}}-\frac{\partial \boldsymbol{L}}{\partial q}=Q_{i} \quad q \propto\left(\theta, \psi, l, h, \frac{,}{2}\right.
$$

Where $Q_{i}$ is the generalized force of the $i^{\text {th }}$ satellite excluding gravity. Using Eqs.(4), (5) and (6) in Eq. (7), we can get the nonlinear equations of motion governing the roll angle $\theta$,the pitch angle $\psi$ and the separation distance $l_{1} 、 l_{2}, l_{3}$.

The 4-craft formation remains statically fixed relative to the frame $\boldsymbol{H}$ in the radial direction, thus, the nonlinear equations satisfy the following equilibrium conditions:

$$
\begin{gathered}
\theta=\dot{\theta}=\ddot{\theta}=\psi=\dot{\psi}=\ddot{\psi}=\dot{l}_{1}=\ddot{l}_{1}=\dot{l}_{2}=\ddot{l}_{2}=\dot{l}_{3}=\ddot{l}_{3}=0 \\
l_{1}=l_{1 f}, l_{2}=l_{2 f}, l_{3}=l_{3 f}
\end{gathered}
$$

The roll and pitch equations of motion are linearized about small roll and pitch angles respectively. In order to get the linearized equations of motion, assuming that the separation distance equations of motion are linearized about small variations $\delta l_{i}$ and so as charge product $\delta Q_{i j}$. Shown as:

$$
\begin{gathered}
l_{1}=l_{1 f}+\delta l_{1}, l_{2}=l_{2 f}+\delta l_{2}, l_{3}=l_{3 f}+\delta l_{3}, \\
Q_{12}=Q_{12 f}+\delta Q_{12}, Q_{13}=Q_{13 f}+\delta Q_{13}, \\
Q_{14}=Q_{14 f}+\delta Q_{14}, Q_{23}=Q_{23 f}+\delta Q_{23}, \\
Q_{24}=Q_{24 f}+\delta Q_{24}, Q_{34}=Q_{34 f}+\delta Q_{34}
\end{gathered}
$$

Where $l_{i f}$ and $Q_{i j f}$ means the reference values. The charge product $Q_{i j}$ should satisfy equations that utilize equilibrium conditions. In this paper, there are an infinite number of charge pairs can satisfy charge product requirements. Performing the necessary linearization and decouple equations about $\delta l_{i}$ yields:

$$
\begin{aligned}
& \ddot{\theta}+4 \Omega^{2} \theta=0 \\
& \ddot{\psi}+3 \Omega^{2} \psi+2 \Omega \frac{X_{1}}{X_{0}} \delta i_{1}+2 \Omega \frac{X_{2}}{X_{0}} \delta i_{2}+2 \Omega \frac{X_{3}}{X_{0}} \delta i_{3}=0 \\
& \delta \ddot{l}_{1}-2 \Omega l_{1 f} \dot{\psi}-3 \Omega^{2} \delta l_{1}-\mu_{1} \frac{m_{1}+m_{2}}{m_{1} m_{2}} \delta Q_{12}+\frac{\mu_{2}}{m_{2}} \delta Q_{23} \\
& -\frac{\mu_{4}}{m_{1}} \delta Q_{13}+\frac{\mu_{5}}{m_{2}} \delta Q_{24}-\frac{\mu_{6}}{m_{1}} \delta Q_{14}=0 \\
& \delta \ddot{l_{2}}-2 \Omega l_{2 f} \dot{\psi}-3 \Omega^{2} \delta l_{2}+\frac{\mu_{1}}{m_{2}} \delta Q_{12}-\mu_{2} \frac{m_{2}+m_{3}}{m_{2} m_{3}} \delta Q_{23}(10) \\
& +\frac{\mu_{3}}{m_{3}} \delta Q_{34}-\frac{\mu_{4}}{m_{3}} \delta Q_{13}-\frac{\mu_{5}}{m_{2}} \delta Q_{24}=0 \\
& \delta \ddot{l_{3}}-2 \Omega l_{3 f} \dot{\psi}-3 \Omega^{2} \delta l_{3}+\frac{\mu_{2}}{m_{3}} \delta Q_{23}-\mu_{3} \frac{m_{3}+m_{4}}{m_{3} m_{4}} \delta Q_{34} \\
& +\frac{\mu_{4}}{m_{3}} \delta Q_{13}-\frac{\mu_{5}}{m_{4}} \delta Q_{24}-\frac{\mu_{6}}{m_{4}} \delta Q_{14}=0 \\
& \text { where } \mu_{1}=\frac{k_{c}}{l_{1 f}^{2}}, \mu_{2}=\frac{k_{c}}{l_{2 f}^{2}}, \mu_{3}=\frac{k_{c}}{l_{3 f}^{2}}, \mu_{4}=\frac{k_{c}}{\left(l_{1 f}+l_{2 f}\right)^{2}}, \\
& \mu_{5}=\frac{k_{c}}{\left(l_{2 f}+l_{3 f}\right)^{2}}, \mu_{6}=\frac{k_{c}}{\left(l_{1 f}+l_{2 f}+l_{3 f}\right)^{2}}, \\
& \mathrm{X}_{0}=a_{1} l_{1 f}^{2}+a_{2} l_{2 f}^{2}+a_{3} l_{3 f}^{2}+2 a_{4} l_{1 f} l_{2 f}+2 a_{5} l_{1 f} l_{3 f}+2 a_{6} l_{2 f} l_{3 f}, \\
& \mathrm{X}_{1}=a_{1} l_{1 f}+a_{4} l_{2 f}+a_{5} l_{3 f}, \mathrm{X}_{2}=a_{2} l_{2 f}+a_{4} l_{1 f}+a_{6} l_{3 f}, \\
& \mathrm{X}_{3}=a_{3} l_{3 f}+a_{5} l_{1 f}+a_{6} l_{2 f}, \Omega \text { is the orbit rate. }
\end{aligned}
$$

\section{Controller Design}

The $\psi 、 \delta l_{i}$ are controlled with Coulomb force and $\theta$ is not controlled with Coulomb force. $\delta Q_{i j}$ is chosen as control variable. Eq.(10) can be represented in the following form:

$$
\dot{x}=A_{1} x+B_{1} u
$$




$$
\boldsymbol{y}=\boldsymbol{C}_{1} \boldsymbol{x}
$$

Where the state $\boldsymbol{x}=\left[\psi, \dot{\psi}, \delta l_{1}, \delta \dot{l}_{1}, \delta l_{2}, \delta \dot{l}_{2}, \delta l_{3}, \delta \dot{l}_{3}\right]^{T}$.Using Coulomb force as an actuator mechanism, the $\boldsymbol{A}_{1} 、 \boldsymbol{B}_{1}$ and $\boldsymbol{C}_{1}$ matrices can be presented from Eq. (10) as:

$$
\begin{gathered}
\boldsymbol{A}_{1}=\left[\begin{array}{cccccccc}
0 & 1 & 0 & 0 & 0 & 0 & 0 & 0 \\
-3 \Omega^{2} & 0 & 0 & -\frac{2 \Omega \xi_{1}}{\xi_{0}} & 0 & -\frac{2 \Omega \xi_{2}}{\xi_{0}} & 0 & -\frac{2 \Omega \xi_{3}}{\xi_{0}} \\
0 & 0 & 0 & 1 & 0 & 0 & 0 & 0 \\
0 & 2 \Omega l_{1 f} & 3 & 0 & 0 & 0 & 0 & 0 \\
0 & 0 & 0 & 0 & 0 & 1 & 0 & 0 \\
0 & 2 \Omega l_{2 f} & 0 & 0 & 3 & 0 & 0 & 0 \\
0 & 0 & 0 & 0 & 0 & 0 & 0 & 1 \\
0 & 2 \Omega l_{3 f} & 0 & 0 & 0 & 0 & 3 & 0
\end{array}\right] \\
\boldsymbol{B}_{1}=\left[\begin{array}{cccccccc}
0 & 0 & & 0 & 0 & 0 & 0 \\
0 & 0 & & 0 & 0 & 0 & 0 \\
\mu_{1} \frac{m_{1}+m_{2}}{m_{1} m_{2}} & -\frac{\mu_{2}}{m_{2}} & & 0 & \frac{\mu_{4}}{m_{1}} & -\frac{\mu_{5}}{m_{2}} & \frac{\mu_{6}}{m_{1}} \\
0 & 0 & & 0 & 0 & 0 & 0 \\
-\frac{\mu_{1}}{m_{2}} & \mu_{2} \frac{m_{2}+m_{3}}{m_{2} m_{3}} & -\frac{\mu_{3}}{m_{3}} & \frac{\mu_{4}}{m_{3}} & \frac{\mu_{5}}{m_{2}} & 0 \\
0 & 0 & & 0 & 0 & 0 & 0 \\
0 & -\frac{\mu_{2}}{m_{3}} & \frac{m_{3}+m_{4}}{m_{3} m_{4}} \mu_{3} & -\frac{\mu_{4}}{m_{3}} & \frac{\mu_{5}}{m_{4}} & \frac{\mu_{6}}{m_{4}}
\end{array}\right] \\
\boldsymbol{C}_{1}=\left[\begin{array}{cccccccc}
1 & 0 & 0 & 0 & 0 & 0 & 0 \\
0 & 0 & 1 & 0 & 0 & 0 & 0 & 0 \\
0 & 0 & 0 & 0 & 1 & 0 & 0 & 0 \\
0 & 0 & 0 & 0 & 0 & 0 & 1 & 0
\end{array}\right]
\end{gathered}
$$

The following feedback control is used to control the system with the feedback gain matrix, $\boldsymbol{K}$, computed using either the pole placement method or the linear quadratic regulator (LQR) method.

$$
\boldsymbol{u}=-\boldsymbol{K} \boldsymbol{x}
$$

Here the LQR methodology is applied to determine the optimal control, $\boldsymbol{u}$, such that the gain vector $\boldsymbol{K}$ minimizes the performance index.

$$
J=\int_{0}^{\infty}\left(\boldsymbol{x}^{T} \boldsymbol{Q} \boldsymbol{x}+\boldsymbol{u}^{T} \boldsymbol{R} \boldsymbol{u}\right) \mathrm{dT}
$$

where $\boldsymbol{u}=\left[\begin{array}{llllll}\delta Q_{12} & \delta Q_{23} & \delta Q_{34} & \delta Q_{13} & \delta Q_{24} & \delta Q_{14}\end{array}\right]^{T}, \quad \boldsymbol{Q}$ and $\boldsymbol{R}$ are weighting matrices used to establish a faster response for control as design parameters.Eq.(10) can also be represented as:

$$
\begin{gathered}
\dot{x}=A_{2} x+B_{2} u+\omega(x) \\
y=C_{2} x
\end{gathered}
$$

where $\boldsymbol{x}=\left[\int \psi, \psi, \dot{\psi}, \int \delta l_{1}, \delta l_{1}, \delta l_{1}, \int \delta l_{2}, \delta l_{2}, \delta i_{2}, \int \delta l_{3}, \delta l_{3}, \delta \dot{l}_{3}\right]^{T}$.

$\boldsymbol{A}_{2} 、 \boldsymbol{B}_{2}$ and $\boldsymbol{C}_{2}$ matrices can be expressed as:

$$
\begin{aligned}
& \boldsymbol{A}_{2}=\left[\begin{array}{cccccccccccc}
0 & 1 & 0 & 0 & 0 & 0 & 0 & 0 & 0 & 0 & 0 & 0 \\
0 & 0 & 1 & 0 & 0 & 0 & 0 & 0 & 0 & 0 & 0 & 0 \\
0 & -3 \Omega^{2} & 0 & 0 & 0 & -\frac{2 \Omega X_{1}}{X_{0}} & 0 & 0 & -\frac{2 \Omega X_{2}}{X_{0}} & 0 & 0 & -\frac{2 \Omega X_{3}}{X_{0}} \\
0 & 0 & 0 & 0 & 1 & 0 & 0 & 0 & 0 & 0 & 0 & 0 \\
0 & 0 & 0 & 0 & 0 & 1 & 0 & 0 & 0 & 0 & 0 & 0 \\
0 & 0 & 2 \Omega l_{1 f} & 0 & 3 & 0 & 0 & 0 & 0 & 0 & 0 & 0 \\
0 & 0 & 0 & 0 & 0 & 0 & 0 & 0 & 1 & 0 & 0 & 0 \\
0 & 0 & 0 & 0 & 0 & 0 & 0 & 0 & 0 & 1 & 0 & 0 \\
0 & 0 & 2 \Omega l_{2 f} & 0 & 0 & 0 & 0 & 3 & 0 & 0 & 0 & 0 \\
0 & 0 & 0 & 0 & 0 & 0 & 0 & 0 & 0 & 0 & 1 & 0 \\
0 & 0 & 0 & 0 & 0 & 0 & 0 & 0 & 0 & 0 & 0 & 1 \\
0 & 0 & 2 \Omega l_{3 f} & 0 & 0 & 0 & 0 & 0 & 0 & 0 & 3 & 0
\end{array}\right] \\
& \boldsymbol{B}_{2}=\left[\begin{array}{cccccc}
0 & 0 & 0 & 0 & 0 & 0 \\
0 & 0 & 0 & 0 & 0 & 0 \\
0 & 0 & 0 & 0 & 0 & 0 \\
0 & 0 & 0 & 0 & 0 & 0 \\
0 & 0 & 0 & 0 & 0 & 0 \\
\mu_{1} \frac{m_{1}+m_{2}}{m_{1} m_{2}} & -\frac{\mu_{2}}{m_{2}} & 0 & \frac{\mu_{4}}{m_{1}} & -\frac{\mu_{5}}{m_{2}} & \frac{\mu_{6}}{m_{1}} \\
0 & 0 & 0 & 0 & 0 & 0 \\
0 & 0 & 0 & 0 & 0 & 0 \\
-\frac{\mu_{1}}{m_{2}} & \mu_{2} \frac{m_{2}+m_{3}}{m_{2} m_{3}} & -\frac{\mu_{3}}{m_{3}} & \frac{\mu_{4}}{m_{3}} & \frac{\mu_{5}}{m_{2}} & 0 \\
0 & 0 & 0 & 0 & 0 & 0 \\
0 & 0 & 0 & 0 & 0 & 0 \\
0 & -\frac{\mu_{2}}{m_{3}} & \frac{m_{3}+m_{4}}{m_{3} m_{4}} & -\frac{\mu_{4}}{m_{3}} & \frac{\mu_{5}}{m_{4}} & \frac{\mu_{6}}{m_{4}}
\end{array}\right] \\
& \boldsymbol{C}_{2}=\left[\begin{array}{llllllllllll}
0 & 1 & 0 & 0 & 0 & 0 & 0 & 0 & 0 & 0 & 0 & 0 \\
0 & 0 & 0 & 0 & 1 & 0 & 0 & 0 & 0 & 0 & 0 & 0 \\
0 & 0 & 0 & 0 & 0 & 0 & 0 & 1 & 0 & 0 & 0 & 0 \\
0 & 0 & 0 & 0 & 0 & 0 & 0 & 0 & 0 & 0 & 1 & 0
\end{array}\right]
\end{aligned}
$$

\section{Numerical simulation}

In this section, we present numerical simulation to illustrate the effectiveness of the proposed LQR controller. In the simulations,

$m_{1}=m_{2}=m_{3}=m_{4}=50 \mathrm{~kg}, l_{1 f}=l_{2 f}=l_{3 f}=20 \mathrm{~m}$,

$k_{c}=8.99 \times 10^{9} \mathrm{Nm}^{2} / \mathrm{C}^{2}, \Omega=2.66 \times 10^{-6} \mathrm{rad} / \mathrm{sec}$,

$\delta \psi(0)=0.1 \mathrm{rad}, \quad \delta l_{1}(0)=\delta l_{2}(0)=\delta l_{3}(0)=0.6 \mathrm{~m}$,

$q_{1}=1.8697 m C, q_{2}=42.445 m C, q_{3}=-46.569 m C$,

$q_{4}=-195.103 m C$.

And in the LQR control, control parameters matrices are:

$$
\begin{aligned}
& \boldsymbol{Q}_{1}=\operatorname{diag}\left[\begin{array}{llllllll}
0.001 & 0.1 & 0.001 & 0.01 & 0.01 & 0.001 & 0.1 & 0.001
\end{array}\right] \\
& \boldsymbol{R}_{1}=\operatorname{diag}\left[\begin{array}{llllll}
10^{8} & 10^{8} & 10^{8} & 10^{7} & 10^{7} & 10^{6}
\end{array}\right] \\
& \boldsymbol{Q}_{2}=\operatorname{diag}\left[\begin{array}{llllllllllll}
1 & 10 & 30 & 0.5 & 0.01 & 0.01 & 0.1 & 0.01 & 0.01 & 0.1 & 0.001 & 0.1
\end{array}\right] \\
& \boldsymbol{R}_{2}=\operatorname{diag}\left[\begin{array}{llllll}
10^{7} & 10^{8} & 10^{7} & 10^{7} & 10^{7} & 10^{7}
\end{array}\right]
\end{aligned}
$$

The feedback gain $\mathrm{K}$ is solved by LQR method of MATLAB.

The dynamics of Coulomb formation system is simulated using the numerical differential equation solver in Matlab/Simulink for several cases. The relative tolerance is $10^{-6}$.

The simulation figures shown as: 


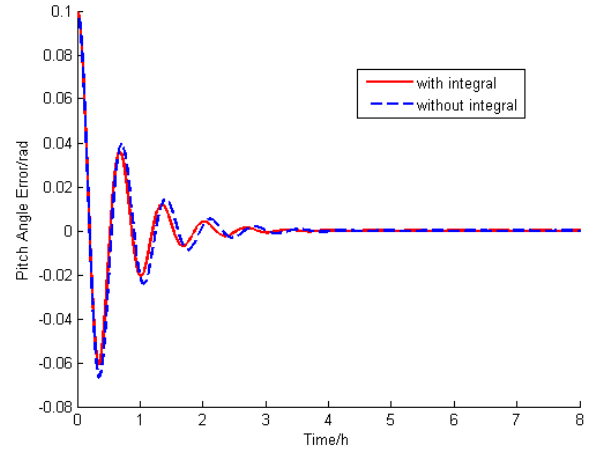

Figure 2. Time history of pitch angle $\delta \psi$

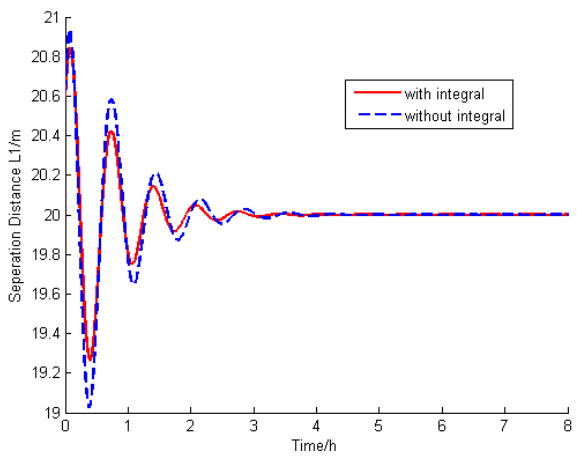

Figure 3. Time history of length variations $l_{1}$

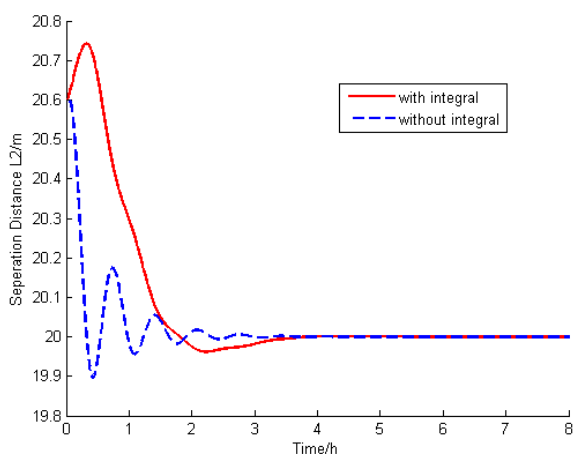

Figure 4. Time history of length variations $l_{2}$

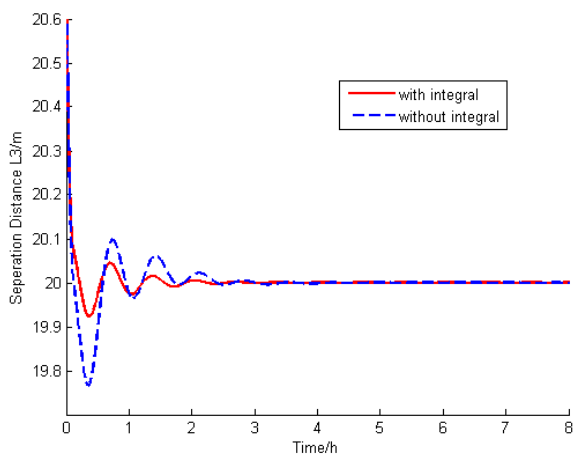

Figure 5. Time history of length variations $l_{3}$

From the simulation results we can see that the pitch angle error (Figure 2) of formation shows a amplitude attenuation and converge to zero at around $4 \mathrm{~h}$. The separation distances (Figure 3 Figure 5) also converge to zero around $4 \mathrm{~h}$. All state variations converge to zero finally with given charge.
In each picture of Figure 2、Figure 3 and Figure 5, curves with improved LQR controller have less amplitude oscillation and converge faster than LQR controller without integral item. In Figure 4, the curve with integral part clearly shows a small amplitude variation and converge to desired distance with less oscillation frequency. It illustrates that the improved LQR controller gets better control results than the traditional LQR controller.

\section{Conclusion}

In this paper, a LQR control law for a 4-craft Coulomb formation at GEO is given. On the basis of dynamics model, considering the model error, the improved LQR control law is designed. Simulation results shows that the improved LQR controller gets better control results in controlling the attitude and separation distance to desired position in finite time. Numerical simulation results demonstrate the validity of the dynamic model and the effectiveness of the proposed control strategy.

\section{References}

1. King, L. B., Parker, G. G., Deshmukh, S., and Chong, J.-H., "Spacecraft Formation-Flying using Inter-Vehicle Coulomb Forces," Tech. rep., NASA/NIAC, January 2002, http://www.niac.usra.edu.

2. King, L. B., Parker, G. G., Deshmukh, S., and Chong, J.-H., "Study of Interspacecraft Coulomb Forces and Implications for Formation Flying," AIAA Journal of Propulsion and Power, Vol. 19, No. 3, May June 2003, pp. 497505.

3. Natarajan A, Schaub H. Linear Dynamics and Stability Analysis of a Coulomb Tether Formation. Journal of Guidance, Control, and Dynamics, 2006, 29(4): 831 839.

4. Natarajan A, Schaub H. Hybrid Control of Orbit Normal and Along-Track Two-Craft Coulomb Tethers. Aerospace Science and Technology, 2009,13:183 191

5. Natarajan A, Schaub H. Orbit-Nadir Aligned Coulomb Tether Reconfiguration Analysis. AAS/AIAA Spaceflight Mechanics Meeting, Galveston, TX, Jan. 27-31, 2008.

6. Inampudi R, Schaub H. Orbit Radial Dynamic Analysis of Two-Craft Coulomb Formation at Libration Points. AAS/AIAA Astrodynamics Specialist Conference, Toronto, Canada, Aug. 25, 2010 .

7. Inampudi $\mathrm{R}$, Schaub $\mathrm{H}$. Orbit-Radial control of Two-Craft Coulomb Formation about circular orbits and Libration Points. 4th International Conference on Spacecraft Formation Flying Missions and Technologies. May 18-20, 2011. 